\title{
Unbounded positive solutions for $m$-point time-scale boundary value problems on infinite intervals
}

\author{
Xiangkui Zhao • Weigao Ge
}

Received: 22 December 2008 / Revised: 21 March 2009 / Published online: 7 April 2009

(C) The Author(s) 2009. This article is published with open access at Springerlink.com

\begin{abstract}
In this work, we consider the following second-order $m$-point boundary value problem on time scales

$$
\left\{\begin{array}{l}
\left(\phi_{p}\left(u^{\triangle}(t)\right)\right)^{\nabla}+h(t) f\left(t, u(t), u^{\triangle}(t)\right)=0, \quad t \in(0,+\infty)_{\mathbb{T}}, \\
u(0)=\sum_{i=1}^{m-2} \alpha_{i} u\left(\eta_{i}\right), \quad u^{\triangle}(+\infty)=\sum_{i=1}^{m-2} \beta_{i} u^{\triangle}\left(\eta_{i}\right) .
\end{array}\right.
$$

We establish new criteria for the existence of at least three unbounded positive solutions. Our results are new even for the corresponding differential $(\mathbb{T}=\mathbb{R})$, difference equation $(\mathbb{T}=\mathbb{Z})$ and for the general time-scale setting. An example is given to illustrate our results.
\end{abstract}

Keywords Time scale $\cdot$ Boundary value problem · Unbounded solution · Fixed point theorem $\cdot$ Weighted Banach space

Mathematics Subject Classification (2000) 39A10

X. Zhao supported by National Natural Science Foundation of China (10671012) and the Doctoral Program Foundation of Education Ministry of China (20050007011).

X. Zhao ( $\varangle)$

Department of Mathematics and Mechanics, School of Applied Science, University of Science and Technology Beijing, Beijing 100083, China

e-mail: zhaoxiangkui@126.com

X. Zhao · W. Ge

Department of Mathematics, Beijing Institute of Technology, Beijing 100081, China 


\section{Introduction}

Calculus on time scales was introduced by Hilger (see [1]), as a theory which includes both differential and difference calculus as special cases. Since then, a large body of theory unifying and generalizing difference and differential equations was developed by Agarwal, Anderson, Bohner, Guseinov, Henderson, Peterson (see [2, 3] and references therein). Further, the study of dynamic equations on time scales has led to several important applications, e.g., insect population models, neural networks, heat transfer and epidemic models (see [2,3] and references therein). Recently, much attention is focused on time-scale boundary value problems on finite intervals (see [4-10] and references therein).

Sun and Wang [8] studied the following three-point boundary value problem on time scales

$$
\left\{\begin{array}{l}
\left(\phi_{p}\left(u^{\triangle}(t)\right)\right)^{\nabla}+h(t) f(t, u(t))=0, \quad t \in(0, T)_{\mathbb{T}}, \\
u(0)-\beta u^{\triangle}(0)=\gamma u^{\triangle}(\eta), \quad u^{\triangle}(T)=0 .
\end{array}\right.
$$

They established sufficient conditions for the existence of at least one, two or three positive solutions.

Karaca [9] studied the following fourth-order four-point boundary value problem on time scales

$$
\left\{\begin{array}{l}
y^{\triangle^{4}}(t)-q(t) y^{\triangle^{2}}(\sigma(t))=f\left(t, y(\sigma(t)), y^{\triangle^{2}}(t)\right), \quad t \in[a, b]_{\mathbb{T}}, \\
y\left(\sigma^{4}(b)\right)=0, \quad \alpha y(a)-\beta y^{\triangle}(a)=0, \\
\gamma y^{\triangle^{2}}\left(\xi_{1}\right)-\delta y^{\triangle^{3}}\left(\xi_{1}\right)=0, \quad \gamma y^{\triangle^{2}}\left(\xi_{2}\right)+\eta y^{\triangle^{3}}\left(\xi_{2}\right)=0 .
\end{array}\right.
$$

They established sufficient conditions for the existence of a positive solution by using Leray-Schauder's fixed point theorem.

Liang, Zhang and Wang [10] studied the following nonlinear $n$ th-order $m$-point singular boundary value problem

$$
\left\{\begin{array}{l}
\left(\phi_{p}\left(u^{\triangle^{n-1}}(t)\right)\right)^{\nabla}+a(t) f\left(t, u(t), u^{\triangle}(t), \ldots, u^{\triangle^{n-2}}(t)\right)=0, \quad t \in(0, T)_{\mathbb{T}}, \\
u^{\triangle^{i}}(0)=0, \quad i=0,1, \ldots, n-3, \\
u^{\triangle^{n-2}}(0)=\sum_{i=1}^{m-2} \alpha_{i} u^{\triangle^{n-2}}\left(\xi_{i}\right), \quad u^{\triangle^{n-1}}(T)=0 .
\end{array}\right.
$$

They established the sufficient conditions for the existence of countably many positive solutions.

Boundary value problems on infinite intervals occur naturally in the study of radially symmetric solutions of nonlinear elliptic equations and various physical phenomena (see [11-16] and references therein). The study of time-scale boundary value problems on infinite intervals was initiated by Agarwal, Bohner and O'Regan [17]. 
The authors studied the following boundary value problems

$$
\left\{\begin{array}{l}
y^{\triangle \triangle}(t)+f(t, y(\sigma(t)))=0, \quad \text { for } t \in[a,+\infty)_{\mathbb{T}}, \\
y(a)=0, \\
y(t) \text { is bounded for } t \in[a,+\infty)_{\mathbb{T}}
\end{array}\right.
$$

and

$$
\left\{\begin{array}{l}
y^{\triangle \Delta}(t)+f(t, y(\sigma(t)))=0, \quad \text { for } t \in[a,+\infty)_{\mathbb{T}}, \\
\alpha y(a)-\beta y^{\triangle}(a)=\gamma, \quad \alpha \geq 0, \beta \geq 0 \text { with } \alpha^{2}+\beta^{2}>0, \\
y(t) \text { is bounded for } t \in[a,+\infty)_{\mathbb{T}} .
\end{array}\right.
$$

They established the sufficient conditions for the existence of a positive solution by using Schauder's fixed point theorem and the operator approximation method. Hao, Liang and Xiao [18] studied the following singular boundary value problem

$$
\left\{\begin{array}{l}
x^{\triangle \Delta}(t)-k^{2} x(\sigma(t))+m(t) f(t, x(\sigma(t)))=0, \quad t \in[0,+\infty) \mathbb{T}, \\
x(0)=0, \quad \lim _{t \rightarrow+\infty} x(t)=0 .
\end{array}\right.
$$

They showed the existence of a positive solution by using Schauder's fixed point theorem and the operator approximation method.

However, there are few literatures available on the existence of unbounded positive solutions for time-scale boundary value problems. Inspired by the mentioned works, we consider the following time-scale boundary value problem

$$
\left\{\begin{array}{l}
\left(\phi_{p}\left(u^{\triangle}(t)\right)\right)^{\nabla}+h(t) f\left(t, u(t), u^{\triangle}(t)\right)=0, \quad t \in(0,+\infty)_{\mathbb{T}}, \\
u(0)=\sum_{i=1}^{m-2} \alpha_{i} u\left(\eta_{i}\right), \quad u^{\triangle}(+\infty)=\sum_{i=1}^{m-2} \beta_{i} u^{\triangle}\left(\eta_{i}\right),
\end{array}\right.
$$

where $u^{\triangle}(+\infty)=\lim _{t \in \mathbb{T}, t \rightarrow+\infty} u^{\triangle}(t), \phi_{p}(s)=|s|^{p-2} s, p>1,\left(\phi_{p}\right)^{-1}=\phi_{q}, \frac{1}{p}+$ $\frac{1}{q}=1, \eta_{1}, \eta_{2}, \ldots, \eta_{m-2} \in \mathbb{T}, \sigma(0)<\eta_{1}<\eta_{2}<\cdots<\eta_{m-2}<+\infty$.

The main features of this paper are as follows. First, comparing with [4-10, 17, 18], we introduce a weighted Banach space so as to overcome the difficulties occurred in the estimation of unbounded solutions. Second, comparing with [4-18], we change the differential equation into a equivalent integral equation by introducing a monotone function because of the nonlinear differential operator $\left(\phi_{p}\left(u^{\Delta}\right)\right)^{\nabla}$ and the boundary condition $u^{\triangle}(+\infty)=\sum_{i=1}^{m-2} \beta_{i} u^{\triangle}\left(\eta_{i}\right)$.

The paper is organized as follows. After this section, some definitions will be presented in Sect. 2. Some lemmas will be established in Sect. 3. Our main results will be given in Sect. 4 .

\section{Preliminary}

Now we list some conditions in this section for convenience.

Let $F(t, u, v)=f\left(t,\left(1+t^{2}\right) u,(1+t) v\right)$. 
(H1) $\sum_{i=1}^{m-2} \alpha_{i}<1, \sum_{i=1}^{m-2} \beta_{i}<1, \alpha_{i} \geq 0, \beta_{i} \geq 0, i=1,2 \ldots, m-2$;

(H2) $h \in C_{l d}\left([0,+\infty)_{\mathbb{T}},[0,+\infty)\right), \int_{0}^{+\infty} h(r) \nabla r<+\infty$;

(H3) $F:[0,+\infty)_{\mathbb{T}} \times[0,+\infty) \times[0,+\infty) \rightarrow[0,+\infty)$;

(H4) For $t \in[0,+\infty)_{\mathbb{T}}, F(t, \cdot, \cdot)$ is continuous;

(H5) For $u, v \in[0,+\infty), F(\cdot, u, v)$ is $l d$-continuous on $\mathbb{T}$;

(H6) When $u, v$ are bounded, $F(t, u, v)$ is bounded.

Throughout the paper, let $\mathbb{T}$ (time scale) be a nonempty closed subset of $\mathbb{R}$ such that $0 \in \mathbb{T}$. We assume that $\mathbb{T}$ has the topology which inherits from the standard topology on $\mathbb{R}$. For $t \in \mathbb{T}$, we define the forward (respectively, backward) jump operator $\sigma: \mathbb{T} \rightarrow \mathbb{T}$ (respectively, $\rho: \mathbb{T} \rightarrow \mathbb{T}$ ) by

$$
\sigma(t)=\inf \{s \in \mathbb{T} \mid s>t\} \quad \text { (respectively, } \rho(t)=\sup \{s \in \mathbb{T} \mid s<t\}) .
$$

In this definition we put $\inf \emptyset=\sup \mathbb{T}$ (i.e., $\sigma(t)=t$ if $\mathbb{T}$ has a maximum $t$ ) and $\sup \emptyset=\inf \mathbb{T}($ i.e., $\rho(t)=t$ if $\mathbb{T}$ has a minimum $t)$, where $\emptyset$ denotes the empty set.

If $a<b$ are points in $\mathbb{T}$, then we let

$$
[a, b]_{\mathbb{T}}=[a, b] \cap \mathbb{T} .
$$

We assume that there exist $t_{n} \in \mathbb{T}, n \in\{1,2, \ldots\} \equiv \mathbb{N}$ with

$$
0<t_{1}<t_{2}<\cdots<t_{n}<\cdots \quad \text { and } \quad t_{n} \uparrow+\infty \quad \text { as } n \rightarrow+\infty \text {. }
$$

Let

$$
[0,+\infty)_{\mathbb{T}}=\bigcup_{n=1}^{+\infty}\left[0, t_{n}\right]_{\mathbb{T}}
$$

Definition 2.1 Let $\mathrm{E}$ be a real Banach space. A nonempty closed set $P \subset E$ is a cone provided that

(1) $a u+b v \in P$ for all $u, v \in P$ and $a \geq 0, b \geq 0$,

(2) $u,-u \in P$ implies $u=0$.

Every cone $P \subset E$ induces an ordering in $E$ given by $x \leq y$ if and only if $y-x \in P$.

Definition 2.2 The map $\alpha$ is a nonnegative continuous concave functional on a cone $P$ of a real Banach space $E$ provided that $\alpha: P \rightarrow[0,+\infty)$ is continuous and

$$
\alpha(t u+(1-t) v) \geq t \alpha(u)+(1-t) \alpha(v)
$$

for all $u, v \in P, 0 \leq t \leq 1$.

Similarly, we say the map $\gamma$ a nonnegative continuous convex functional on a cone $P$ of a real Banach space $E$ provided that

$$
\gamma(t u+(1-t) v) \leq t \gamma(u)+(1-t) \gamma(v)
$$


for all $u, v \in P, 0 \leq t \leq 1$.

Let $\gamma$ and $\theta$ be nonnegative continuous convex functionals on a cone $P$, $\alpha$ be nonnegative continuous concave functional on $P$ and $\psi$ be nonnegative continuous functional on $P$. Then for positive real numbers $a, b, c$ and $d$, we define the following convex sets

$$
\begin{aligned}
& P(\gamma, d)=\{u \in P \mid \gamma(u)<d\}, \\
& P(\gamma, \alpha, b, d)=\{u \in P \mid b \leq \alpha(u), \gamma(u) \leq d\}, \\
& P(\gamma, \theta, \alpha, b, c, d)=\{u \in P \mid b \leq \alpha(u), \theta(u) \leq c, \gamma(u) \leq d\}, \\
& R(\gamma, \psi, a, d)=\{u \in P \mid a \leq \psi(u), \gamma(u) \leq d\}
\end{aligned}
$$

Theorem 2.1 ([3]) Let $P$ be a cone in a real Banach space E. Assume that there exist two positive numbers $M$ and $d$, two nonnegative continuous convex functionals $\gamma$ and $\theta$ on $P$, a nonnegative continuous concave functional $\alpha$ on $P$ and a nonnegative continuous functional $\psi$ on $P$ such that $\psi(\lambda u) \leq \lambda \psi(u)$ for all $0 \leq \lambda \leq 1$ and

$$
\alpha(u) \leq \psi(u), \quad\|u\| \leq M \gamma(u)
$$

for all $u \in \overline{P(\gamma, d)}$. Suppose that $T: \overline{P(\gamma, d)} \rightarrow \overline{P(\gamma, d)}$ is completely continuous and there exist three positive numbers $a, b$ and $c$ with $a<b$ such that

(S1) $\{u \in P(\gamma, \theta, \alpha, b, c, d) \mid \alpha(u)>b\} \neq \emptyset$ and $\alpha(T u)>b$ for $u \in P(\gamma, \theta, \alpha, b, c, d)$; (S2) $\alpha(T u)>b$ for $u \in P(\gamma, \alpha, b, d)$ with $\theta(T u)>c$;

(S3) $0 \notin R(\gamma, \psi, a, d)$ and $\psi(T u)<a$ for $u \in R(\gamma, \psi, a, d)$ with $\psi(T u)=a$.

Then $T$ has at least three fixed points $u_{1}, u_{2}, u_{3} \in \overline{P(\gamma, d)}$ such that

$$
\gamma\left(u_{i}\right) \leq d, \quad i=1,2,3, \quad \psi\left(u_{1}\right)<a, \quad a<\psi\left(u_{2}\right) \text { with } \alpha\left(u_{2}\right)<b, \alpha\left(u_{3}\right)>b .
$$

Definition 2.3 Assume that $y: \mathbb{T} \rightarrow \mathbb{R}$ is a function. Let $t \in \mathbb{T}^{k}$, then we define $y^{\Delta}(t)$ to be the number (provided it exists) with the property that given any $\varepsilon>0$, there is a neighborhood $U$ of $t$ (i.e., $U=(t-\delta, t+\delta) \cap \mathbb{T}$ for some $\delta>0)$ such that

$$
\left|(y(\sigma(t))-y(s))-y^{\triangle}(t)(\sigma(t)-s)\right| \leq \varepsilon|\sigma(t)-s|
$$

for all $s \in U$. We call $y^{\triangle}(t)$ the delta (or Hilger) derivative of $y$ at $t$.

Definition 2.4 Assume that $y: \mathbb{T} \rightarrow \mathbb{R}$ is a function. Let $t \in \mathbb{T}_{k}$, then we define $y^{\nabla}(t)$ to be the number (provided it exists) with the property that given any $\varepsilon>0$, there is a neighborhood $U$ of $t$ such that

$$
\left|(y(\rho(t))-y(s))-y^{\nabla}(t)(\rho(t)-s)\right| \leq \varepsilon|\rho(t)-s|
$$

for all $s \in U$. We call $y^{\nabla}(t)$ the nabla derivative of $y$ at $t$. 


\section{Related lemmas}

Lemma 3.1 Suppose that $y \in C_{l d}[0,+\infty)_{\mathbb{T}}$ and $\lim _{t \in \mathbb{T}, t \rightarrow+\infty} y(t)$ exists, then $y$ is bounded on $[0,+\infty)_{\mathbb{T}}$.

Proof Assume that $y:[0,+\infty)_{\mathbb{T}} \rightarrow \mathbb{R}$ is unbounded, i.e., for each $n \in \mathbb{N}$, there exists a sequence $\left\{t_{n}\right\}$ with $t_{n} \in[0,+\infty)_{\mathbb{T}}$ and $\left|y\left(t_{n}\right)\right|>n$. Since

$$
\left\{t_{n} \mid n \in \mathbb{N}\right\} \subset[0,+\infty)_{\mathbb{T}},
$$

there exists a subsequence $\left\{t_{n_{k}}\right\}_{k \in \mathbb{N}}$ such that

$$
\lim _{k \rightarrow+\infty} t_{n_{k}}=t_{0}<+\infty
$$

or

$$
t_{n_{k}} \uparrow+\infty \quad \text { as } k \rightarrow+\infty .
$$

Since $\lim _{t \in \mathbb{T}, t \rightarrow+\infty} y(t)$ exists, we get $\lim _{k \rightarrow+\infty} t_{n_{k}}=t_{0}<+\infty$. Note that $t_{0} \in \mathbb{T}$, since $\mathbb{T}$ is closed. Hence there exists either a subsequence that tends to $t_{0}$ from above or a subsequence that tends to $t_{0}$ from below. In any case, the limit of $y$ as $t \rightarrow t_{0}$ has to be finite according to $l d$-continuous, a contradiction. The proof is complete.

Consider the space $E$ defined by

$$
E=\left\{u \in C_{l d}^{\triangle}[0,+\infty)_{\mathbb{T}}\left|\sup _{t \in[0,+\infty)_{\mathbb{T}}}\right| \frac{u(t)}{1+t^{2}} \mid<+\infty, \lim _{t \in \mathbb{T}, t \rightarrow+\infty} \frac{u^{\triangle}(t)}{1+t} \text { exists }\right\}
$$

with the norm

$$
\|u\|=\max \left\{\sup _{t \in[0,+\infty)_{\mathbb{T}}}\left|\frac{u(t)}{1+t^{2}}\right|, \sup _{t \in[0,+\infty)_{\mathbb{T}}}\left|\frac{u^{\triangle}(t)}{1+t}\right|\right\} .
$$

Using standard arguments, we can obtain that $(E,\|\cdot\|)$ is a Banach space. Define the cone $P \subset E$ by

$$
\begin{gathered}
P=\left\{u \in E \mid u(0)=\sum_{i=1}^{m-2} \alpha_{i} u\left(\eta_{i}\right), u\right. \text { is nondecreasing, nonnegative and concave on } \\
\left.[0,+\infty)_{\mathbb{T}}\right\} .
\end{gathered}
$$

Define the operator $T: P \rightarrow E$ by

$$
\begin{aligned}
(T u)(t)= & \frac{\sum_{i=1}^{m-2} \alpha_{i} \int_{0}^{\eta_{i}} \phi_{q}\left(A_{u}+\int_{s}^{+\infty} h(r) f\left(r, u(r), u^{\triangle}(r)\right) \nabla r\right) \Delta s}{1-\sum_{i=1}^{m-2} \alpha_{i}} \\
& +\int_{0}^{t} \phi_{q}\left(A_{u}+\int_{s}^{+\infty} h(r) f\left(r, u(r), u^{\triangle}(r)\right) \nabla r\right) \triangle s,
\end{aligned}
$$


where

$$
\phi_{q}\left(A_{u}\right)=\sum_{i=1}^{m-2} \beta_{i} \phi_{q}\left(A_{u}+\int_{\eta_{i}}^{+\infty} h(r) f\left(r, u(r), u^{\triangle}(r)\right) \nabla r\right) .
$$

Lemma 3.2 If (H1), (H3) hold, then for $u \in C_{l d}^{\triangle}[0,+\infty)_{\mathbb{T}}$, there exists a unique $A_{u} \in(-\infty,+\infty)$ satisfying (3.4).

Proof For $u \in C_{l d}^{\triangle}[0,+\infty) \mathbb{T}$, define

$$
H_{u}(c)=\phi_{q}(c)-\sum_{i=1}^{m-2} \beta_{i} \phi_{q}\left(c+\int_{\eta_{i}}^{+\infty} h(r) f\left(r, u(r), u^{\triangle}(r)\right) \nabla r\right) .
$$

Then $H_{u}(c) \in C((-\infty,+\infty), R)$. By (H1), (H3) we get $H_{u}(0) \leq 0$. We will consider two cases to prove $H_{u}(c)=0$ has a unique solution on $(-\infty,+\infty)$, which means there exists a unique $A_{u} \in(-\infty,+\infty)$ satisfying (3.4).

Case 1: $H_{u}(0)=0$.

By $H_{u}(0)=0$, we get

$$
\sum_{i=1}^{m-2} \beta_{i} \phi_{q}\left(\int_{\eta_{i}}^{+\infty} h(r) f\left(r, u(r), u^{\triangle}(r)\right) \nabla r\right)=0 .
$$

Hence

$$
\beta_{i} \phi_{q}\left(\int_{\eta_{i}}^{+\infty} h(r) f\left(r, u(r), u^{\triangle}(r)\right) \nabla r\right)=0 \quad(i=1,2, \ldots, m-2),
$$

i.e.,

$$
\phi_{p}\left(\beta_{i}\right) \int_{\eta_{i}}^{+\infty} h(r) f\left(r, u(r), u^{\triangle}(r)\right) \nabla r=0 \quad(i=1,2, \ldots, m-2) .
$$

Then

$$
\begin{aligned}
H_{u}(c) & =\phi_{q}(c)-\sum_{i=1}^{m-2} \beta_{i} \phi_{q}\left(c+\int_{\eta_{i}}^{+\infty} h(r) f\left(r, u(r), u^{\triangle}(r)\right) \nabla r\right) \\
& =\phi_{q}(c)-\sum_{i=1}^{m-2} \phi_{q}\left(\phi_{p}\left(\beta_{i}\right) c+\phi_{p}\left(\beta_{i}\right) \int_{\eta_{i}}^{+\infty} h(r) f\left(r, u(r), u^{\triangle}(r)\right) \nabla r\right) \\
& =\phi_{q}(c)-\sum_{i=1}^{m-2} \beta_{i} \phi_{q}(c)=\left(1-\sum_{i=1}^{m-2} \beta_{i}\right) \phi_{q}(c) .
\end{aligned}
$$

Hence we get there exists a unique $c=0$ satisfying $H_{u}(c)=0$. 
Case 2: $H_{u}(0) \neq 0$, i.e., $H_{u}(0)<0$.

(i) When $c \in(-\infty, 0)$

$$
\begin{aligned}
H_{u}(c) & =\phi_{q}(c)-\sum_{i=1}^{m-2} \beta_{i} \phi_{q}\left(c+\int_{\eta_{i}}^{+\infty} h(r) f\left(r, u(r), u^{\triangle}(r)\right) \nabla r\right) \\
& \leq \phi_{q}(c)-\sum_{i=1}^{m-2} \beta_{i} \phi_{q}(c) \\
& =\left(1-\sum_{i=1}^{m-2} \beta_{i}\right) \phi_{q}(c)<0 .
\end{aligned}
$$

So when $c \in(-\infty, 0), H_{u}(c) \neq 0$.

(ii) When $c \in(0,+\infty)$

$$
\begin{aligned}
H_{u}(c) & =\phi_{q}(c)-\sum_{i=1}^{m-2} \beta_{i} \phi_{q}\left(c+\int_{\eta_{i}}^{+\infty} h(r) f\left(r, u(r), u^{\triangle}(r)\right) \nabla r\right) \\
& =\phi_{q}(c)\left(1-\sum_{i=1}^{m-2} \beta_{i} \phi_{q}\left(1+\frac{\int_{\eta_{i}}^{+\infty} h(r) f\left(r, u(r), u^{\triangle}(r)\right) \nabla r}{c}\right)\right) \\
& =\phi_{q}(c) \bar{H}(c)
\end{aligned}
$$

where

$$
\bar{H}_{u}(c)=1-\sum_{i=1}^{m-2} \beta_{i} \phi_{q}\left(1+\frac{\int_{\eta_{i}}^{+\infty} h(r) f\left(r, u(r), u^{\triangle}(r)\right) \nabla r}{c}\right) .
$$

Since $H_{u}(0)<0$, that is $\sum_{i=1}^{m-2} \beta_{i} \phi_{q}\left(\int_{\eta_{i}}^{+\infty} h(r) f\left(r, u(r), u^{\triangle}(r)\right) \nabla r\right)>0$. As a result, there must exist $i_{0} \in\{1,2, \ldots, n\}$ such that $\beta_{i_{0}} \phi_{q}\left(\int_{\eta_{i_{0}}}^{+\infty} h(r) f\left(r, u(r), u^{\triangle}(r)\right) \nabla r\right)$ $>0$. Thus, we get $\bar{H}_{u}(c)$ is strictly increasing on $(0,+\infty), \int_{0}^{+\infty} h(r) f(r, u(r)$, $\left.u^{\triangle}(r)\right) \nabla r>0$ and $\sum_{i=1}^{m-2} \beta_{i}>0$. Let

$$
\bar{c}=\frac{\phi_{p}\left(\sum_{i=1}^{m-2} \beta_{i}\right)}{1-\phi_{p}\left(\sum_{i=1}^{m-2} \beta_{i}\right)} \int_{0}^{+\infty} h(r) f\left(r, u(r), u^{\triangle}(r)\right) \nabla r,
$$

then $\bar{c}>0$ and we have

$$
\begin{aligned}
\bar{H}_{u}(\bar{c}) & =1-\sum_{i=1}^{m-2} \beta_{i} \phi_{q}\left(1+\frac{\left(1-\phi_{p}\left(\sum_{i=1}^{m-2} \beta_{i}\right)\right) \int_{\eta_{i}}^{+\infty} h(r) f\left(r, u(r), u^{\triangle}(r)\right) \nabla r}{\phi_{p}\left(\sum_{i=1}^{m-2} \beta_{i}\right) \int_{0}^{+\infty} h(r) f\left(r, u(r), u^{\triangle}(r)\right) \nabla r}\right) \\
& \geq 1-\sum_{i=1}^{m-2} \beta_{i} \phi_{q}\left(1+\frac{1-\phi_{p}\left(\sum_{i=1}^{m-2} \beta_{i}\right)}{\phi_{p}\left(\sum_{i=1}^{m-2} \beta_{i}\right)}\right)
\end{aligned}
$$




$$
\begin{aligned}
& =1-\sum_{i=1}^{m-2} \beta_{i} \phi_{q}\left(\frac{1}{\phi_{p}\left(\sum_{i=1}^{m-2} \beta_{i}\right)}\right) \\
& =0 .
\end{aligned}
$$

So $H_{u}(\bar{c})=\phi_{q}(\bar{c}) \bar{H}(\bar{c}) \geq 0$. The mean value theorem guarantees that there exists a $c_{0} \in(0, \bar{c}] \subset(0,+\infty)$ such that $H_{u}\left(c_{0}\right)=0$. If there exist two constants $c_{i} \in(-\infty, 0)$ $(i=1,2)$ satisfying $H_{u}\left(c_{1}\right)=H_{u}\left(c_{2}\right)=0$, then $\bar{H}_{u}\left(c_{1}\right)=\bar{H}_{u}\left(c_{2}\right)=0$. Since $\bar{H}_{u}(c)$ is strictly increasing on $(0,+\infty)$, we get $c_{1}=c_{2}$. Therefore $H_{u}(c)=0$ has a unique solution on $(0,+\infty)$.

Combining (i), (ii) and $H_{u}(0) \neq 0$, we obtain that $H_{u}(c)=0$ has a unique solution on $(-\infty,+\infty)$. The proof is complete.

Remark 3.1 From the proof of Lemma 3.2, we know that for $u \in C_{l d}^{\triangle}[0,+\infty)_{\mathbb{T}}$,

$$
A_{u} \in\left[0, \frac{\phi_{p}\left(\sum_{i=1}^{m-2} \beta_{i}\right)}{1-\phi_{p}\left(\sum_{i=1}^{m-2} \beta_{i}\right)} \int_{0}^{+\infty} h(r) f\left(r, u(r), u^{\triangle}(r)\right) \nabla r\right] .
$$

Moreover, if $H_{u}(0)=0$, then $A_{u}=0$; if $H_{u}(0) \neq 0$, then $A_{u} \neq 0$.

The boundary value problem (1.1) has a solution $u=u(t)$, if and only if $u$ solves the operator equation $u=T u$. Since the Arzela-Ascoli theorem fails to work in the space $E$, we need a modified compactness criterion to prove that $T$ is compact. Let the nonnegative continuous concave functional $\alpha$, the nonnegative continuous convex functionals $\gamma, \theta$ and the nonnegative continuous functional $\psi$ be defined on the cone $P$ by

$$
\begin{gathered}
\gamma(u)=\sup _{t \in[0,+\infty)_{\mathbb{T}}} \frac{u^{\triangle}(t)}{1+t}, \\
\psi(u)=\theta(u)=\sup _{t \in[0,+\infty)_{\mathbb{T}}} \frac{u(t)}{1+t^{2}}, \\
\alpha(u)=\frac{k^{2}}{k^{2}+1} \min _{t \in\left[\frac{1}{k^{2}}, k^{2}\right]_{\mathbb{T}}} u(t)=\frac{k^{2}}{k^{2}+1} u\left(\frac{1}{k^{2}}\right)
\end{gathered}
$$

for $u \in P$, where $\frac{1}{k^{2}} \in \mathbb{T}, \frac{1}{k^{2}}<\sigma\left(\eta_{1}\right)$ and $k^{2}>\max \left\{1, \xi_{m-2}\right\}$.

Lemma 3.3 If (H1) holds, then $\sup _{t \in[0,+\infty)_{\mathbb{T}}} \frac{u(t)}{1+t^{2}} \leq M \sup _{t \in[0,+\infty)_{\mathbb{T}}} \frac{u^{\Delta}(t)}{1+t}$ for $u \in P$, where $M=1+\frac{\sum_{i=1}^{m-2} \alpha_{i} \eta_{i}}{1-\sum_{i=1}^{m-2} \alpha_{i}}$.

Proof For $u \in P$, one arrives at $u\left(\eta_{i}\right)-u(0) \leq \eta_{i} u^{\triangle}(0)$. Hence

$$
\sum_{i=1}^{m-2} \alpha_{i} u\left(\eta_{i}\right)-\sum_{i=1}^{m-2} \alpha_{i} u(0) \leq \sum_{i=1}^{m-2} \alpha_{i} \eta_{i} u^{\triangle}(0) .
$$


By

$$
u(0)=\sum_{i=1}^{m-2} \alpha_{i} u\left(\eta_{i}\right)
$$

we get

$$
u(0) \leq \frac{\sum_{i=1}^{m-2} \alpha_{i} \eta_{i}}{1-\sum_{i=1}^{m-2} \alpha_{i}} u^{\triangle}(0)
$$

Hence

$$
\begin{aligned}
\frac{u(t)}{1+t^{2}} & =\frac{1}{1+t^{2}}\left(\int_{0}^{t} u^{\triangle}(s) \triangle s+u(0)\right) \\
& \leq \frac{1}{1+t^{2}}\left(t u^{\triangle}(0)+\frac{\sum_{i=1}^{m-2} \alpha_{i} \eta_{i}}{1-\sum_{i=1}^{m-2} \alpha_{i}} u^{\triangle}(0)\right) \\
& <\left(1+\frac{\sum_{i=1}^{m-2} \alpha_{i} \eta_{i}}{1-\sum_{i=1}^{m-2} \alpha_{i}}\right) u^{\triangle}(0) \\
& =M u^{\triangle}(0),
\end{aligned}
$$

i.e.,

$$
\sup _{t \in[0,+\infty)_{\mathbb{T}}} \frac{u(t)}{1+t^{2}} \leq M u^{\triangle}(0)=M \sup _{t \in[0,+\infty)_{\mathbb{T}}} \frac{u^{\triangle}(t)}{1+t} .
$$

The proof is complete.

From Lemma 3.3, we obtain

$$
\begin{aligned}
\|u\| & =\max \left\{\sup _{t \in[0,+\infty)_{\mathbb{T}}}\left|\frac{u(t)}{1+t^{2}}\right|, \sup _{t \in[0,+\infty)_{\mathbb{T}}}\left|\frac{u^{\triangle}(t)}{1+t}\right|\right\} \\
& \leq \max \left\{M \sup _{t \in[0,+\infty)_{\mathbb{T}}}\left|\frac{u^{\triangle}(t)}{1+t}\right|, \sup _{t \in[0,+\infty)_{\mathbb{T}}}\left|\frac{u^{\triangle}(t)}{1+t}\right|\right\} \\
& =M \gamma(u) .
\end{aligned}
$$

Therefore the condition (2.1) of Theorem 2.1 is satisfied.

Lemma 3.4 If $u \in P$, then $\alpha(u) \geq \frac{1}{k^{2}+1} \theta(u)$.

Proof Since $u$ is concave and nonnegative,

$$
\begin{aligned}
\alpha(u) & =\frac{k^{2}}{k^{2}+1} u\left(\frac{1}{k^{2}}\right) \\
& =\frac{k^{2}}{k^{2}+1} u\left(\frac{k^{2}+k^{2} t^{2}-1}{k^{2}+k^{2} t^{2}} \cdot \frac{1+t^{2}-t}{k^{2}+k^{2} t^{2}-1}+\frac{t}{k^{2}+k^{2} t^{2}}\right)
\end{aligned}
$$




$$
\begin{aligned}
& >\frac{k^{2}}{k^{2}+1} \frac{u(t)}{k^{2}+k^{2} t^{2}} \\
& =\frac{1}{k^{2}+1} \frac{u(t)}{1+t^{2}}
\end{aligned}
$$

for $u \in P, t \in[0,+\infty)_{\mathbb{T}}$. Hence $\alpha(u) \geq \frac{1}{k^{2}+1} \sup _{t \in[0,+\infty)_{\mathbb{T}}} \frac{u(t)}{1+t^{2}}=\frac{1}{1+k^{2}} \theta(u)$. The proof is complete.

Now for convenience, we introduce the following notation. Let

$$
\omega=\int_{0}^{+\infty} h(r) \nabla r, \quad \Lambda=\frac{\omega \phi_{p}\left(\sum_{i=1}^{m-2} \beta_{i}\right)}{1-\phi_{p}\left(\sum_{i=1}^{m-2} \beta_{i}\right)}
$$

Lemma 3.5 For $u \in C_{l d}^{\triangle}[0,+\infty)_{\mathbb{T}}$, let $A_{u}$ satisfy (3.4) corresponding to u. Suppose that (H1)-(H6) hold, then $A_{u}: C_{l d}^{\triangle}[0,+\infty)_{\mathbb{T}} \rightarrow R$ is continuous about $u$.

Proof Suppose $\left\{u_{n}\right\} \subset C_{l d}^{\triangle}[0,+\infty)_{\mathbb{T}}$ with $u_{n} \rightarrow u_{0} \in C_{l d}^{\triangle}[0,+\infty)_{\mathbb{T}}$, then there exists $r_{0}$ such that

$$
\max \left\{\|u\|, \sup _{n \in N \backslash\{0\}}\left\|u_{n}\right\|\right\}<r_{0} .
$$

Let $A_{n}(n=0,1,2, \ldots)$ be constants decided by (3.4) corresponding to $u_{n}$ ( $n=$ $0,1,2, \ldots)$. By (H6), we get that $F(t, u, v)$ is bounded on $[0,+\infty)_{\mathbb{T}} \times\left[0, r_{0}\right]^{2}$. Set

$$
B_{0}=\sup \left\{F(t, u, v) \mid(t, u, v) \in[0,+\infty) \mathbb{T} \times\left[0, r_{0}\right]^{2}\right\} .
$$

Since

$$
\begin{gathered}
\frac{\phi_{p}\left(\sum_{i=1}^{m-2} \beta_{i}\right)}{1-\phi_{p}\left(\sum_{i=1}^{m-2} \beta_{i}\right)} \int_{0}^{+\infty} h(r) f\left(r, u(r), u^{\triangle}(r)\right) \nabla r \\
=\frac{\phi_{p}\left(\sum_{i=1}^{m-2} \beta_{i}\right)}{1-\phi_{p}\left(\sum_{i=1}^{m-2} \beta_{i}\right)} \int_{0}^{+\infty} h(r) F\left(r, \frac{u(r)}{1+r^{2}}, \frac{u^{\triangle}(r)}{1+r}\right) \nabla r \\
\leq \frac{B_{0} \phi_{p}\left(\sum_{i=1}^{m-2} \beta_{i}\right)}{1-\phi_{p}\left(\sum_{i=1}^{m-2} \beta_{i}\right)} \int_{0}^{+\infty} h(r) \nabla r \\
=B_{0} \Lambda, \\
A_{n} \in\left[0, \frac{\phi_{p}\left(\sum_{i=1}^{m-2} \beta_{i}\right)}{1-\phi_{p}\left(\sum_{i=1}^{m-2} \beta_{i}\right)} \int_{0}^{+\infty} h(r) f\left(r, u(r), u^{\triangle}(r)\right) \nabla r\right] \subseteq\left[0, B_{0} \Lambda\right],
\end{gathered}
$$

which means $\left\{A_{n}\right\}$ is bounded.

Suppose that sequence $\left\{A_{n}\right\}$ does not converge, then there exist two subsequences $\left\{A_{n_{k}}^{(1)}\right\}$ and $\left\{A_{n_{k}}^{(2)}\right\}$ of $\left\{A_{n}\right\}$ with $A_{n_{k}}^{(1)} \rightarrow c_{1}, A_{n_{k}}^{(2)} \rightarrow c_{2}$ and $c_{1} \neq c_{2}$. Combining (H4) 
and using the Lebesgue's dominated convergence theorem, we get

$$
\begin{aligned}
\phi_{q}\left(c_{1}\right) & =\lim _{n_{k} \rightarrow+\infty} \sum_{i=1}^{m-2} \beta_{i} \phi_{q}\left(A_{n_{k}}^{(1)}+\int_{\eta_{i}}^{+\infty} h(r) f\left(r, u_{n_{k}}(r), u_{n_{k}}^{\triangle}(r)\right) \nabla r\right) \\
& =\sum_{i=1}^{m-2} \beta_{i} \phi_{q}\left(\lim _{n_{k} \rightarrow+\infty} A_{n_{k}}^{(1)}+\lim _{n_{k} \rightarrow+\infty} \int_{\eta_{i}}^{+\infty} h(r) f\left(r, u_{n_{k}}(r), u_{n_{k}}^{\Delta}(r)\right) \nabla r\right) \\
& =\sum_{i=1}^{m-2} \beta_{i} \phi_{q}\left(c_{1}+\int_{\eta_{i}}^{+\infty} h(r) f\left(r, u_{0}(r), u_{0}^{\triangle}(r)\right) \nabla r\right) .
\end{aligned}
$$

Since sequence $\left\{A_{n}\right\}$ is unique, we get $c_{1}=A_{0}$. Similarly $c_{2}=A_{0}$. So $c_{1}=c_{2}$, which is a contradiction. Therefore $A_{n} \rightarrow A_{0}$ for $u_{n} \rightarrow u_{0}$, which means $A_{u}$ : $C_{l d}^{\triangle}[0,+\infty)_{\mathbb{T}} \rightarrow R$ is continuous. The proof is complete.

Lemma 3.6 Suppose that $(\mathrm{H} 1)-(\mathrm{H} 6)$ hold, then $T: P \rightarrow P$ is completely continuous.

Proof We divide the proof into four steps.

Step 1: We show that $T P \subset P$.

For $u \in P$, by $(\mathrm{H} 1)-(\mathrm{H} 3)$, we have

$$
\begin{aligned}
& \sum_{i=1}^{m-2} \alpha_{i}(T u)\left(\eta_{i}\right) \\
& =\sum_{i=1}^{m-2} \alpha_{i}\left(\frac{\sum_{i=1}^{m-2} \alpha_{i} \int_{0}^{\eta_{i}} \phi_{q}\left(A_{u}+\int_{s}^{+\infty} h(r) f\left(r, u(r), u^{\triangle}(r)\right) \nabla r\right) \Delta s}{1-\sum_{i=1}^{m-2} \alpha_{i}}\right) \\
& \quad+\sum_{i=1}^{m-2} \alpha_{i} \int_{0}^{\eta_{i}} \phi_{q}\left(A_{u}+\int_{s}^{+\infty} h(r) f\left(r, u(r), u^{\triangle}(r)\right) \nabla r\right) \triangle s \\
& =\frac{\sum_{i=1}^{m-2} \alpha_{i} \int_{0}^{\eta_{i}} \phi_{q}\left(A_{u}+\int_{s}^{+\infty} h(r) f\left(r, u(r), u^{\triangle}(r)\right) \nabla r\right) \triangle s}{1-\sum_{i=1}^{m-2} \alpha_{i}} \\
& =(T u)(0), \\
& (T u)(t)=\frac{\sum_{i=1}^{m-2} \alpha_{i} \int_{0}^{\eta_{i}} \phi_{q}\left(A_{u}+\int_{s}^{+\infty} h(r) f\left(r, u(r), u^{\triangle}(r)\right) \nabla r\right) \Delta s}{1-\sum_{i=1}^{m-2} \alpha_{i}} \\
& \quad+\int_{0}^{t} \phi_{q}\left(A_{u}+\int_{s}^{+\infty} h(r) f\left(r, u(r), u^{\triangle}(r)\right) \nabla r\right) \triangle s \\
& (T u)^{\triangle}(t)=0, \phi_{q}\left(A_{u}+\int_{t}^{+\infty} h(r) f\left(r, u(r), u^{\triangle}(r)\right) \nabla r\right) \geq 0 .
\end{aligned}
$$


If $t \in[0,+\infty)_{\mathbb{T}}$ is left scattered, then

$$
(T u)^{\Delta \nabla}(t)=\frac{(T u)^{\triangle}(t)-(T u)^{\triangle}(\rho(t))}{t-\rho(t)} \leq 0 \quad \text { on }[0,+\infty)_{\mathbb{T}} .
$$

If $t \in[0,+\infty)_{\mathbb{T}}$ is left dense, then

$$
(T u)^{\Delta \nabla}(t)=\lim _{s \rightarrow t} \frac{(T u)^{\triangle}(t)-(T u)^{\triangle}(s)}{t-s} \leq 0 \quad \text { on }[0,+\infty)_{\mathbb{T}} .
$$

Hence $T u$ is negative, concave and nondecreasing on $[0,+\infty)_{\mathbb{T}}$, i.e., $T P \subset P$.

Step 2: We show that $T: P \rightarrow P$ is continuous.

Let $u_{n} \rightarrow u$ as $n \rightarrow+\infty$ in $P$, then there exists $r_{0}$ such that

$$
\max \left\{\|u\|, \sup _{n \in N \backslash\{0\}}\left\|u_{n}\right\|\right\}<r_{0} .
$$

By (H6), we get that $F(t, u, v)$ is bounded on $[0,+\infty) \mathbb{T} \times\left[0, r_{0}\right]^{2}$. Set

$$
B_{0}=\sup \left\{F(t, u, v),(t, u, v) \in[0,+\infty)_{\mathbb{T}} \times\left[0, r_{0}\right]^{2}\right\} .
$$

We get

$$
\begin{aligned}
& \left|\phi_{p}\left(\left(T u_{n}\right)^{\triangle}(t)\right)-\phi_{p}\left((T u)^{\triangle}(t)\right)\right| \\
& \quad \leq\left|A_{n}-A_{u}\right|+\int_{t}^{+\infty} h(r)\left|f\left(r, u_{n}(r), u_{n}^{\triangle}(r)\right)-f\left(r, u(r), u^{\triangle}(r)\right)\right| \nabla r \\
& \quad=\left|A_{n}-A_{u}\right|+\int_{t}^{+\infty} h(r)\left|F\left(r, \frac{u_{n}(r)}{1+r^{2}}, \frac{u_{n}^{\triangle}(r)}{1+r}\right)-F\left(r, \frac{u(r)}{1+r^{2}}, \frac{u^{\triangle}(r)}{1+r}\right)\right| \nabla r \\
& \quad \leq 2 B_{0}+2 B_{0} \omega .
\end{aligned}
$$

Hence

$$
\sup _{t \in[0,+\infty)_{\mathbb{T}}}\left|\frac{\left(T u_{n}\right)^{\triangle}(t)-(T u)^{\triangle}(t)}{1+t}\right| \rightarrow 0 \quad \text { as } n \rightarrow+\infty
$$

Since

$$
\begin{gathered}
A_{u}+\int_{0}^{+\infty} h(r) f\left(r, u(r), u^{\triangle}(r)\right) \nabla r \\
\leq B_{0} \Lambda+\int_{0}^{+\infty} h(r) F\left(r, \frac{u(r)}{1+r^{2}}, \frac{u^{\triangle}(r)}{1+r}\right) \nabla r \\
\leq B_{0}(\Lambda+\omega), \\
\sup _{t \in[0,+\infty)_{\mathbb{T}}} \frac{1}{1+t^{2}}\left|\left(T u_{n}\right)(t)-(T u)(t)\right| \\
\leq \sup _{t \in[0,+\infty)_{\mathbb{T}}} \frac{1}{1+t^{2}}\left(\frac{\sum_{i=1}^{m-2} \alpha_{i}}{1-\sum_{i=1}^{m-2} \alpha_{i}} \int_{0}^{\eta_{i}} \mid \phi_{q}\left(A_{n}\right.\right.
\end{gathered}
$$




$$
\begin{aligned}
& \left.+\int_{s}^{+\infty} h(r) f\left(r, u_{n}(r), u_{n}^{\triangle}(r)\right) \nabla r\right) \\
& -\phi_{q}\left(A_{u}+\int_{s}^{+\infty} h(r) f\left(r, u(r), u^{\triangle}(r)\right) \nabla r\right) \mid \triangle s \\
& +\int_{0}^{t} \mid \phi_{q}\left(A_{n}+\int_{s}^{+\infty} h(r) f\left(r, u_{n}(r), u_{n}^{\triangle}(r)\right) \nabla r\right) \\
& \left.-\phi_{q}\left(A_{u}+\int_{s}^{+\infty} h(r) f\left(r, u(r), u^{\triangle}(r)\right) \nabla r\right) \mid \triangle s\right) \\
& \leq \sup _{t \in[0,+\infty)_{\mathbb{T}}} \frac{(q-1)\left(B_{0}(\Lambda+\omega)\right)^{q-2}}{1+t^{2}}\left(\frac { \sum _ { i = 1 } ^ { m - 2 } \alpha _ { i } } { 1 - \sum _ { i = 1 } ^ { m - 2 } \alpha _ { i } } \int _ { 0 } ^ { \eta _ { i } } \left(\left|A_{n}-A_{u}\right|\right.\right. \\
& \left.+\int_{s}^{+\infty} h(r)\left|f\left(r, u_{n}(r), u_{n}^{\triangle}(r)\right)-f\left(r, u(r), u^{\triangle}(r)\right)\right| \nabla r\right) \triangle s \\
& +\int_{0}^{t}\left(\left|A_{n}-A_{u}\right|\right. \\
& \left.\left.+\int_{s}^{+\infty} h(r)\left|f\left(r, u_{n}(r), u_{n}^{\triangle}(r)\right)-f\left(r, u(r), u^{\triangle}(r)\right)\right| \nabla r\right) \triangle s\right) \\
& \leq \sup _{t \in[0,+\infty)_{\mathbb{T}}} \frac{(q-1)\left(B_{0}(\Lambda+\omega)\right)^{q-2}}{1+t^{2}}\left(\frac { \sum _ { i = 1 } ^ { m - 2 } \alpha _ { i } } { 1 - \sum _ { i = 1 } ^ { m - 2 } \alpha _ { i } } \int _ { 0 } ^ { \eta _ { i } } \left(\left|A_{n}-A_{u}\right|\right.\right. \\
& \left.+\int_{s}^{+\infty} h(r)\left|F\left(r, \frac{u_{n}(r)}{1+r^{2}}, \frac{u_{n}^{\triangle}(r)}{1+r}\right)-F\left(r, \frac{u(r)}{1+r^{2}}, \frac{u^{\triangle}(r)}{1+r}\right)\right| \nabla r\right) \triangle s \\
& +\int_{0}^{t}\left(\left|A_{n}-A_{u}\right|\right. \\
& \left.\left.+\int_{s}^{+\infty} h(r)\left|F\left(r, \frac{u_{n}(r)}{1+r^{2}}, \frac{u_{n}^{\Delta}(r)}{1+r}\right)-F\left(r, \frac{u(r)}{1+r^{2}}, \frac{u^{\triangle}(r)}{1+r}\right)\right| \nabla r\right) \triangle s\right) \\
& \leq 2(q-1)\left(B_{0}(\Lambda+\omega)\right)^{q-1}\left(1+\frac{\sum_{i=1}^{m-2} \alpha_{i} \eta_{i}}{1-\sum_{i=1}^{m-2} \alpha_{i}}\right) \text {. }
\end{aligned}
$$

By (H4), we get

$$
\sup _{t \in[0,+\infty)_{\mathbb{T}}}\left|\frac{\left(T u_{n}\right)(t)-(T u)(t)}{1+t^{2}}\right| \rightarrow 0 \quad \text { as } n \rightarrow+\infty .
$$

Hence

$$
\begin{aligned}
\left\|T u_{n}-T u\right\|=\max \left\{\sup _{t \in[0,+\infty)_{\mathbb{T}}}\left|\frac{\left(T u_{n}\right)(t)-(T u)(t)}{1+t^{2}}\right|,\right. \\
\left.\sup _{t \in[0,+\infty)_{\mathbb{T}}}\left|\frac{\left(T u_{n}\right)^{\triangle}(t)-(T u)^{\triangle}(t)}{1+t}\right|\right\} \rightarrow 0,
\end{aligned}
$$

as $n \rightarrow+\infty$. 
Hence $T: P \rightarrow P$ is continuous.

Step 3: We show that $T: P \rightarrow P$ is relatively compact.

Let $\Omega$ be any bounded subset of $P$, then there exists $L>0$ such that $\|u\| \leq L$. Set

$$
B_{L}=\sup \left\{F(t, u, v),(t, u, v) \in[0,+\infty)_{\mathbb{T}} \times[0, L]^{2}\right\} .
$$

For $u \in \Omega$, we have

$$
\begin{aligned}
\|T u\| & =\max \left\{\sup _{t \in[0,+\infty)_{\mathbb{T}}}\left|\frac{(T u)(t)}{1+t^{2}}\right|, \sup _{t \in[0,+\infty)_{\mathbb{T}}}\left|\frac{(T u)^{\triangle}(t)}{1+t}\right|\right\} \\
& \leq M(T u)^{\triangle}(0) \\
& \leq M \phi_{q}\left(A_{u}+\int_{0}^{+\infty} h(r) f\left(r, u(r), u^{\triangle}(r)\right) \nabla r\right) \\
& =M \phi_{q}\left(A_{u}+\int_{0}^{+\infty} h(r) F\left(r, \frac{u(r)}{1+r^{2}}, \frac{u^{\triangle}(r)}{1+r}\right) \nabla r\right) \\
& \leq M \phi_{q}\left(B_{L} \Lambda+B_{L} \omega\right) .
\end{aligned}
$$

Hence $T \Omega$ is uniformly bounded.

Now we show that $(T \Omega)^{\triangle}$ is locally equicontinuous on $[0,+\infty)_{\mathbb{T}}$. For any $v>0$, $t_{1}, t_{2} \in[0, v]_{\mathbb{T}}$ and $u \in \Omega$, without loss of generality, we may assume that $t_{2}>t_{1}$.

$$
\begin{aligned}
& \left|\phi_{p}\left((T u)^{\triangle}\left(t_{1}\right)\right)-\phi_{p}\left((T u)^{\triangle}\left(t_{2}\right)\right)\right| \\
& \quad=\int_{t_{1}}^{t_{2}} h(r) F\left(r, \frac{u(r)}{1+r^{2}}, \frac{u^{\triangle}(r)}{1+r}\right) \nabla r \\
& \quad \leq \phi_{q}\left(B_{L}\right) \int_{t_{1}}^{t_{2}} h(r) \nabla r .
\end{aligned}
$$

Hence $\left|\frac{(T u)^{\triangle}\left(t_{1}\right)-(T u)^{\triangle}\left(t_{2}\right)}{1+t}\right| \rightarrow 0$ as $t_{1} \rightarrow t_{2}$. This implies that $(T \Omega)^{\triangle}$ is equicontinuous on $[0, v]_{\mathbb{T}}$. Since $v$ is arbitrary, $(T \Omega)^{\triangle}$ is locally equicontinuous on $[0,+\infty)_{\mathbb{T}}$.

Step 4: We show that $T: P \rightarrow P$ is equiconvergent at $+\infty$.

For $u \in \Omega$, we have

$$
\begin{aligned}
|(T u)(t)|= & \frac{\sum_{i=1}^{m-2} \alpha_{i} \int_{0}^{\eta_{i}} \phi_{q}\left(A_{u}+\int_{s}^{+\infty} h(r) f\left(r, u(r), u^{\triangle}(r)\right) \nabla r\right) \Delta s}{1-\sum_{i=1}^{m-2} \alpha_{i}} \\
& +\int_{0}^{t} \phi_{q}\left(A_{u}+\int_{s}^{+\infty} h(r) f\left(r, u(r), u^{\triangle}(r)\right) \nabla r\right) \triangle s \\
= & \frac{\sum_{i=1}^{m-2} \alpha_{i} \int_{0}^{\eta_{i}} \phi_{q}\left(A_{u}+\int_{s}^{+\infty} h(r) F\left(r, \frac{u(r)}{1+r^{2}}, \frac{u^{\Delta}(r)}{1+r}\right) \nabla r\right) \triangle s}{1-\sum_{i=1}^{m-2} \alpha_{i}} \\
& +\int_{0}^{t} \phi_{q}\left(A_{u}+\int_{s}^{+\infty} h(r) F\left(r, \frac{u(r)}{1+r^{2}}, \frac{u^{\triangle}(r)}{1+r}\right) \nabla r\right) \triangle s
\end{aligned}
$$




$$
\leq \frac{\sum_{i=1}^{m-2} \alpha_{i} \eta_{i} \phi_{q}\left(B_{L}(\Lambda+\omega)\right)}{1-\sum_{i=1}^{m-2} \alpha_{i}}+\phi_{q}\left(B_{L}(\Lambda+\omega)\right) t
$$

Hence

$$
\lim _{t \in \mathbb{T}, t \rightarrow+\infty} \frac{(T u)(t)}{1+t^{2}}=0 .
$$

From

$$
\left|\phi_{p}\left((T u)^{\triangle}\left(t_{1}\right)\right)-\phi_{p}\left((T u)^{\triangle}\left(t_{2}\right)\right)\right| \leq \phi_{q}\left(B_{L}\right) \int_{t_{1}}^{t_{2}} h(r) \nabla r,
$$

we have

$$
\lim _{t \in \mathbb{T}, t \rightarrow+\infty} \frac{(T u)^{\triangle}(t)}{1+t}=0 .
$$

Therefore $T: P \rightarrow P$ is equiconvergent at $+\infty$. From steps $1-4$ together with Lemma 3.1, we get $T: P \rightarrow P$ is completely continuous. The proof is complete.

\section{Existence of three unbounded solutions}

We are ready to apply Avery-Peterson's fixed point theorem to give sufficient conditions for the existence of at least three positive solutions to the boundary value problem (1.1). Now for convenience, we introduce the following notation. Let

$$
\begin{aligned}
& \Upsilon=\phi_{q}\left(\frac{\omega}{1-\phi_{p}\left(\sum_{i=1}^{m-2} \beta_{i}\right)}\right), \\
& N=\phi_{q}\left(\int_{\frac{1}{k^{2}}}^{k^{2}} h(r) \nabla r\right), \\
& c=b\left(k^{2}+1\right)+\frac{\sum_{i=1}^{m-2} \alpha_{i} \eta_{i}}{1-\sum_{i=1}^{m-2} \alpha_{i}} b\left(k^{2}+1\right) .
\end{aligned}
$$

Theorem 4.1 Assume (H1)-(H6) hold. Let $0<a<b \leq \frac{d}{k^{2}+1}$ and suppose that $F$ satisfies the following conditions

(A1) $F(t, u, v) \leq \phi_{p}(d / \Upsilon)$ for $(t, u, v) \in[0,+\infty) \mathbb{T} \times[0, M d] \times[0, d]$;

(A2) $F(t, u, v)>\phi_{p}\left(\left(k^{2}+1\right) b / N\right)$ for $(t, u, v) \in\left[\frac{1}{k^{2}}, k^{2}\right]_{\mathbb{T}} \times\left[\frac{b}{k^{2}}, c\right] \times[0, d]$;

(A3) $F(t, u, v)<\phi_{p}(a / M \Upsilon)$ for $(t, u, v) \in[0,+\infty)_{\mathbb{T}} \times[0, a] \times[0, d]$.

Then the boundary value problem (1.1) has at least three positive solutions $u_{1}, u_{2}$ and $u_{3}$ such that

$$
\sup _{t \in[0,+\infty)_{\mathbb{T}}} \frac{u_{i}^{\triangle}(t)}{1+t} \leq d, \quad i=1,2,3,
$$




$$
\begin{aligned}
& \sup _{t \in[0,+\infty)_{\mathbb{T}}} \frac{u_{1}(t)}{1+t^{2}}<a, \quad a<\sup _{t \in[0,+\infty)_{\mathbb{T}}} \frac{u_{2}(t)}{1+t^{2}}<c \text { with } \min _{t \in\left[\frac{1}{k^{2}}, k^{2}\right] \mathbb{T}}\left|u_{2}(t)\right|<\frac{k^{2}+1}{k^{2}} b, \\
& \sup _{t \in[0,+\infty)_{\mathbb{T}}} \frac{u_{3}(t)}{1+t^{2}}<M d \quad \text { with } \min _{t \in\left[\frac{1}{k^{2}}, k^{2}\right]_{\mathbb{T}}}\left|u_{3}(t)\right|>\frac{k^{2}+1}{k^{2}} b .
\end{aligned}
$$

Proof The boundary value problem (1.1) has a solution $u=u(t)$ if and only if $u$ solves the operator equation $u=T u$. Thus we set out to verify that the operator $T$ satisfies Avery -Peterson's fixed point theorem which will prove the existence of three fixed points of $T$. Now the proof is divided into four steps.

Step 1: We will show that (A1) implies that

$$
T: \overline{P(\gamma, d)} \rightarrow \overline{P(\gamma, d)}
$$

In fact, for $u \in \overline{P(\gamma, d)}$, there is $\gamma(u)=\sup _{t \in[0,+\infty)_{\mathbb{T}}} \frac{u^{\Delta}(t)}{1+t} \leq d$. From Lemma 3.3,

$$
\sup _{t \in[0,+\infty)_{\mathbb{T}}} \frac{u(t)}{1+t^{2}} \leq M d,
$$

then condition (A1) implies

$$
F\left(t, \frac{u(t)}{1+t^{2}}, \frac{u^{\triangle}(t)}{1+t}\right) \leq \phi_{p}(d / \Upsilon) .
$$

On the other hand, for $u \in P$, there is $T u \in P$, then $T u$ is nonnegative, concave and nondecreasing on $[0,+\infty)_{\mathbb{T}}$, so

$$
\begin{aligned}
\gamma(T u)= & \sup _{t \in[0,+\infty)_{\mathbb{T}}} \frac{(T u)^{\triangle}(t)}{1+t}=(T u)^{\triangle}(0) \\
= & \phi_{q}\left(A_{u}+\int_{t}^{+\infty} h(r) f\left(r, u(r), u^{\triangle}(r)\right) \nabla r\right) \\
\leq & \phi_{q}\left(\frac{\phi_{p}\left(\sum_{i=1}^{m-2} \beta_{i}\right)}{1-\phi_{p}\left(\sum_{i=1}^{m-2} \beta_{i}\right)} \int_{0}^{+\infty} h(r) f\left(r, u(r), u^{\triangle}(r)\right) \nabla r\right. \\
& \left.+\int_{t}^{+\infty} h(r) f\left(r, u(r), u^{\triangle}(r)\right) \nabla r\right) \\
= & \phi_{q}\left(\frac{\phi_{p}\left(\sum_{i=1}^{m-2} \beta_{i}\right)}{1-\phi_{p}\left(\sum_{i=1}^{m-2} \beta_{i}\right)} \int_{0}^{+\infty} h(r) F\left(r, \frac{u(r)}{1+r^{2}}, \frac{u^{\triangle}(r)}{1+r}\right) \nabla r\right. \\
& \left.+\int_{t}^{+\infty} h(r) F\left(r, \frac{u(r)}{1+r^{2}}, \frac{u^{\triangle}(r)}{1+r}\right) \nabla r\right) \\
\leq & \frac{d}{\Upsilon} \phi_{q}\left(\frac{\phi_{p}\left(\sum_{i=1}^{m-2} \beta_{i}\right)}{1-\phi_{p}\left(\sum_{i=1}^{m-2} \beta_{i}\right)} \int_{0}^{+\infty} h(r) \nabla r+\int_{0}^{+\infty} h(r) \nabla r\right)
\end{aligned}
$$




$$
\begin{aligned}
& =\frac{d}{\Upsilon} \phi_{q}\left(\frac{\omega}{1-\phi_{p}\left(\sum_{i=1}^{m-2} \beta_{i}\right)}\right) \\
& =d .
\end{aligned}
$$

Thus (4.1) holds.

Step 2: We show that condition (S1) in Theorem 2.1 holds.

We take $u(t)=b\left(k^{2}+1\right) t+\frac{\sum_{i=1}^{m-2} \alpha_{i} \eta_{i}}{1-\sum_{i=1}^{m-2} \alpha_{i}} b\left(k^{2}+1\right)$ for $t \in[0,+\infty)_{\mathbb{T}}$. By (3.5), we get

$$
\begin{aligned}
& \gamma(u)=b\left(k^{2}+1\right)<d, \\
& \theta(u)=\sup _{t \in[0,+\infty)_{\mathbb{T}}} \frac{u(t)}{1+t^{2}} \leq \frac{1}{2} b\left(k^{2}+1\right)+\frac{\sum_{i=1}^{m-2} \alpha_{i} \eta_{i}}{1-\sum_{i=1}^{m-2} \alpha_{i}} b\left(k^{2}+1\right)<c, \\
& \alpha(u)=\frac{k^{2}}{1+k^{2}} u\left(\frac{1}{k^{2}}\right)=\frac{k^{2}}{k^{2}+1}\left(\frac{b\left(k^{2}+1\right)}{k^{2}}+\frac{\sum_{i=1}^{m-2} \alpha_{i} \eta_{i}}{1-\sum_{i=1}^{m-2} \alpha_{i}} b\left(k^{2}+1\right)\right)>b .
\end{aligned}
$$

Hence $\{u \in P(\gamma, \theta, \alpha, b, c, d) \mid \alpha(u)>b\} \neq \emptyset$. Thus for $u \in P(\gamma, \theta, \alpha, b, c, d)$, we get

$$
\frac{b}{k^{2}} \leq \frac{u(t)}{1+t^{2}} \leq c, 0 \leq \frac{u^{\triangle}(t)}{1+t} \leq d \quad \text { for } t \in\left[\frac{1}{k^{2}}, k^{2}\right]_{\mathbb{T}} .
$$

By condition (A2), we get

$$
F\left(t, \frac{u(t)}{1+t^{2}}, \frac{u^{\triangle}(t)}{1+t}\right)>\phi_{p}\left(\left(k^{2}+1\right) b / N\right) \quad \text { for } t \in\left[\frac{1}{k^{2}}, k^{2}\right]_{\mathbb{T}} .
$$

By (A2), we have

$$
\begin{aligned}
\alpha(T u) & =\frac{k^{2}}{k^{2}+1} \min _{t \in\left[\frac{1}{k^{2}}, k^{2}\right]_{\mathbb{T}}}|(T u)(t)|=\frac{k^{2}}{k^{2}+1}(T u)\left(\frac{1}{k^{2}}\right) \\
& \geq \frac{k^{2}}{k^{2}+1} \int_{0}^{\frac{1}{k^{2}}} \phi_{q}\left(\int_{s}^{+\infty} h(r) f\left(r, u(r), u^{\triangle}(r)\right) \nabla r\right) \triangle s \\
& \geq \frac{1}{k^{2}+1} \phi_{q}\left(\int_{\frac{1}{k^{2}}}^{+\infty} h(r) f\left(r, u(r), u^{\triangle}(r)\right) \nabla r\right) \\
& \geq \frac{1}{k^{2}+1} \phi_{q}\left(\int_{\frac{1}{k^{2}}}^{k^{2}} h(r) f\left(r, u(r), u^{\triangle}(r)\right) \nabla r\right) \\
& =\frac{1}{k^{2}+1} \phi_{q}\left(\int_{\frac{1}{k^{2}}}^{k^{2}} h(r) F\left(r, \frac{u(r)}{1+r^{2}}, \frac{u^{\triangle}(r)}{1+r}\right) \nabla r\right) \\
& >\frac{1}{k^{2}+1} \phi_{q}\left(\int_{\frac{1}{k^{2}}}^{k^{2}} h(r) \phi_{p}\left(\frac{k^{2}+1}{N} b\right) \nabla r\right)
\end{aligned}
$$




$$
=\frac{1}{k^{2}+1} \frac{\left(k^{2}+1\right) b}{N} \phi_{q}\left(\int_{\frac{1}{k^{2}}}^{k^{2}} h(r) \nabla r\right)=b .
$$

Therefore we have

$$
\alpha(T u)>b \quad \text { for all } u \in P(\gamma, \theta, \alpha, b, c, d) .
$$

Consequently, condition (S1) in Theorem 2.1 is satisfied.

Step 3: We now prove that (S2) in Theorem 2.1 holds. By Lemma 3.4, we have

$$
\alpha(T u) \geq \frac{1}{k^{2}+1} \theta(T u)>\frac{1}{k^{2}+1} c=\frac{1}{k^{2}+1}\left(b\left(k^{2}+1\right)+\frac{\sum_{i=1}^{m-2} \alpha_{i} \eta_{i}}{1-\sum_{i=1}^{m-2} \alpha_{i}} b\left(k^{2}+1\right)\right) \geq b
$$

for $u \in P(\gamma, \alpha, b, d)$ with $\theta(T u)>c$. Hence condition (S2) in Theorem 2.1 is satisfied.

Step 4: Finally, we prove that (S3) in Theorem 2.1 is satisfied. Since $\psi(0)=0<a$, $0 \notin R(\gamma, \psi, a, d)$. Suppose that $u \in R(\gamma, \psi, a, d)$ with $\psi(u)=a$, then by (A3), we get

$$
\begin{aligned}
\psi(T u)= & \sup _{t \in[0,+\infty)_{\mathbb{T}}} \frac{(T u)^{\triangle}(t)}{1+t} \\
\leq & M(T u)^{\triangle}(0) \\
\leq & M \phi_{q}\left(\frac{\phi_{p}\left(\sum_{i=1}^{m-2} \beta_{i}\right)}{1-\phi_{p}\left(\sum_{i=1}^{m-2} \beta_{i}\right)} \int_{0}^{+\infty} h(r) f\left(r, u(r), u^{\triangle}(r)\right) \nabla r\right. \\
& \left.+\int_{0}^{+\infty} h(r) f\left(r, u(r), u^{\triangle}(r)\right) \nabla r\right) \\
= & M \phi_{q}\left(\frac{\phi_{p}\left(\sum_{i=1}^{m-2} \beta_{i}\right)}{1-\phi_{p}\left(\sum_{i=1}^{m-2} \beta_{i}\right)} \int_{0}^{+\infty} h(r) F\left(r, \frac{u(r)}{1+r^{2}}, \frac{u^{\triangle}(r)}{1+r}\right) \nabla r\right. \\
& \left.+\int_{0}^{+\infty} h(r) F\left(r, \frac{u(r)}{1+r^{2}}, \frac{u^{\triangle}(r)}{1+r}\right) \nabla r\right) \\
\leq & M \frac{a}{M \Upsilon} \phi_{q}\left(\frac{\phi_{p}\left(\sum_{i=1}^{m-2} \beta_{i}\right)}{1-\phi_{p}\left(\sum_{i=1}^{m-2} \beta_{i}\right)} \int_{0}^{+\infty} h(r) \nabla r+\int_{0}^{+\infty} h(r) \nabla r\right) \\
= & M \frac{a}{M \Upsilon} \phi_{q}\left(\frac{\omega}{1-\phi_{p}\left(\sum_{i=1}^{m-2} \beta_{i}\right)}\right)=a .
\end{aligned}
$$

Thus condition (S3) in Theorem 2.1 holds. From steps 1-4 together with Theorem 2.1, we get that the boundary value problem (1.1) has at least three positive solutions $u_{1}, u_{2}, u_{3}$ such that

$$
\sup _{t \in[0,+\infty)_{\mathbb{T}}} \frac{u_{i}^{\Delta}(t)}{1+t} \leq d, \quad i=1,2,3,
$$


$\sup _{t \in[0,+\infty)_{\mathbb{T}}} \frac{u_{1}(t)}{1+t^{2}}<a, \quad a<\sup _{t \in[0,+\infty)_{\mathbb{T}}} \frac{u_{2}(t)}{1+t^{2}}<c$ with $\min _{t \in\left[\frac{1}{k^{2}}, k^{2}\right]_{\mathbb{T}}}\left|u_{2}(t)\right|<\frac{k^{2}+1}{k^{2}} b$, $\sup _{t \in[0,+\infty)_{\mathbb{T}}} \frac{u_{3}(t)}{1+t^{2}}<M d \quad$ with $\min _{t \in\left[\frac{1}{k^{2}}, k^{2}\right]_{\mathbb{T}}}\left|u_{3}(t)\right|>\frac{k^{2}+1}{k^{2}} b$.

The proof is complete.

Example 1 Let $\mathbb{T}=[0,5] \cup\{6,7,8,9\} \cup[10,+\infty), p=3, m=4, \eta_{1}=\frac{1}{3}, \eta_{2}=$ $\frac{2}{3}, \alpha_{1}=\alpha_{2}=\beta_{1}=\beta_{2}=\frac{1}{3}, M=2$ in the boundary value problem (1.1). Now we consider the following problem

$$
\left\{\begin{array}{l}
\left(\left|u^{\triangle}\right| u^{\triangle}\right)^{\nabla}(t)+f\left(t, u(t), u^{\triangle}(t)\right)=0, \quad t \in(0,+\infty)_{\mathbb{T}}, \\
u(0)=\frac{1}{3} u\left(\frac{1}{3}\right)+\frac{1}{3} u\left(\frac{2}{3}\right), \quad u^{\triangle}(+\infty)=\frac{1}{3} u^{\triangle}\left(\frac{1}{3}\right)+\frac{1}{3} u^{\triangle}\left(\frac{2}{3}\right),
\end{array}\right.
$$

where

$$
\begin{gathered}
h(t)=e^{-t}, \\
F(t, u, v)= \begin{cases}\frac{t}{1+t^{2}}\left(2 \times 10^{5} u^{10}+\frac{1}{100}\left(\frac{v}{2 \times 10^{3}}\right)^{4}\right), & u \leq 1,0 \leq v, t \in \mathbb{T} \\
\frac{t}{1+t^{2}}\left(2 \times 10^{5}+\frac{1}{100}\left(\frac{v}{2 \times 10^{3}}\right)^{4}\right), & u>1,0 \leq v, t \in \mathbb{T} .\end{cases}
\end{gathered}
$$

Choose $a=\frac{1}{10}, b=5, k=2, d=2 \times 10^{3}$. We note $\Upsilon \leq 2, C=50, N=\sqrt{\frac{e \frac{15}{4}-1}{e^{4}}}$. Consequently, $F(t, u, v)$ satisfies

(1) $F(t, u, v)<10^{6}<\phi_{p}(d / \Upsilon)$ for $(t, u, v) \in[0,+\infty)_{\mathbb{T}} \times\left[0,4 \times 10^{3}\right] \times[0,2 \times$ $\left.10^{3}\right]$

(2) $F(t, u, v)>4 \times 10^{4}>\phi_{p}\left(\left(k^{2}+1\right) b / N\right)$ for $(t, u, v) \in\left[\frac{1}{4}, 4\right]_{\mathbb{T}} \times\left[\frac{5}{4}, 50\right] \times[0,2 \times$ $\left.10^{3}\right]$

(3) $F(t, u, v)<10^{-5}<\phi_{p}(a / M \Upsilon)$ for $(t, u, v) \in[0,+\infty)_{\mathbb{T}} \times\left[0, \frac{1}{10}\right] \times\left[0,2 \times 10^{3}\right]$.

Then all conditions of Theorem 4.1 hold. From Theorem 4.1, we get that the boundary value problem (4.2) has at least three positive solutions $u_{1}, u_{2}, u_{3}$ such that

$$
\begin{aligned}
& \sup _{t \in[0,+\infty)_{\mathbb{T}}} u_{i}^{\Delta}(t) \leq 2 \times 10^{3}, \quad i=1,2,3, \\
& \sup _{t \in[0,+\infty)_{\mathbb{T}}} \frac{u_{1}(t)}{1+t^{2}}<\frac{1}{10}, \\
& \frac{1}{10}<\sup _{t \in[0,+\infty)_{\mathbb{T}}} \frac{u_{2}(t)}{1+t^{2}}<50 \quad \text { with } \min _{t \in\left[\frac{1}{k^{2}}, k^{2}\right]_{\mathbb{T}}}\left|u_{2}(t)\right|<\frac{25}{4}, \\
& \sup _{t \in[0,+\infty)_{\mathbb{T}}} \frac{u_{3}(t)}{1+t^{2}}<4 \times 10^{3} \quad \text { with } \min _{t \in\left[\frac{1}{k^{2}}, k^{2}\right]_{\mathbb{T}}}\left|u_{3}(t)\right|>\frac{25}{4} .
\end{aligned}
$$


Open Access This article is distributed under the terms of the Creative Commons Attribution Noncommercial License which permits any noncommercial use, distribution, and reproduction in any medium, provided the original author(s) and source are credited.

\section{References}

1. Hilger, S.: Analysis on measure chains a unified approach to continuous and discrete calculus. Results Math. 18, 18-56 (1990)

2. Bohner, M., Peterson, A.: Dynamic Equations on Time Scales: An Introduction with Application. Birkhäuser, Boston (2001)

3. Bohner, M., Peterson, A.: Advances in Dynamic Equations on Time Scales. Birkhäuser, Boston (2003)

4. Zhang, Z., Dong, W., Li, Q., Liang, H.: Existence of nonoscillatory solutions for higher order neutral dynamic equations on time scales. J. Appl. Math. Comput. 28, 29-38 (2008)

5. Rchid, S.A.M., Torres, D.F.M.: Existence of positive solutions for non local $p$-Laplacian thermistor problems on time scales. J. Inequal. Pure Appl. Math. 8, 1-10 (2007)

6. Rchid, S.A.M., Torres, D.F.M.: Existence of infinitely many solutions for a quasilinear elliptic problem on time scales. Int. J. Pure Appl. Math. 39, 239-248 (2007)

7. Feng, M., Zhang, X., Ge, W.: Positive solutions for a class of boundary value problems on time scales. Comput. Math. Appl. 54, 467-475 (2007)

8. Sun, H., Wang, Y.: Existence of positive solutions for $p$-Laplacian three-point boundary-value problems on time scales. Electron. J. Differ. Equ. 92, 1-14 (2008)

9. Karaca, I.Y.: Fourth-order four-point boundary value problem on time scales. Appl. Math. Lett. 21, $1057-1063$ (2008)

10. Liang, S., Zhang, J., Wang, Z.: Existence of countably many positive solutions for $n$ th-order $m$-point boundary-value problems on time scales. Electron. J. Differ. Equ. 123, 1-13 (2008)

11. Baxley, J.V.: Existence and uniqueness of nonlinear boundary value problems on infinite intervals. J. Math. Anal. Appl. 147, 127-133 (1990)

12. Kawano, N., Yanagida, E., Yotsutani, S.: Structure theorems for positive radial solutions to $\Delta u+$ $K(|x|) u^{p}=0$ in $R^{n}$. Funkcial. Ekvac. 36, 557-579 (1993)

13. Aronson, D., Crandall, M.G., Peletier, L.A.: Stabilization of solutions of a degenerate nonlinear diffusion problem. Nonlinear Anal. 6, 1001-1022 (1982)

14. Iffland, G.: Positive solutions of a problem Emden-Fowler type with a type free boundary. SIAM J. Math. Anal. 18, 283-292 (1987)

15. Brighi, B., Hoernel, J.: Asymptotic behavior of the unbounded solutions of some boundary layer equations. Arch. Math. 85, 161-166 (2005)

16. Yan, B., O’Regan, D., Agarwal, R.P.: Unbounded solutions for singular boundary value problems on the semi-infinite interval: Upper and lower solutions and multiplicity. J. Comput. Appl. Math. 197, 365-386 (2006)

17. Agarwal, R.P., Bohner, M., O'Regan, D.: Time scale boundary value problems on infinite intervals. J. Comput. Appl. Math. 141, 27-34 (2002)

18. Hao, Z.C., Liang, J., Xiao, T.J.: Singular boundary value problem on infinite time scale. Discrete Dyn. Nat. Soc. (2006). doi:10.1155/DDNS/2006/71580 